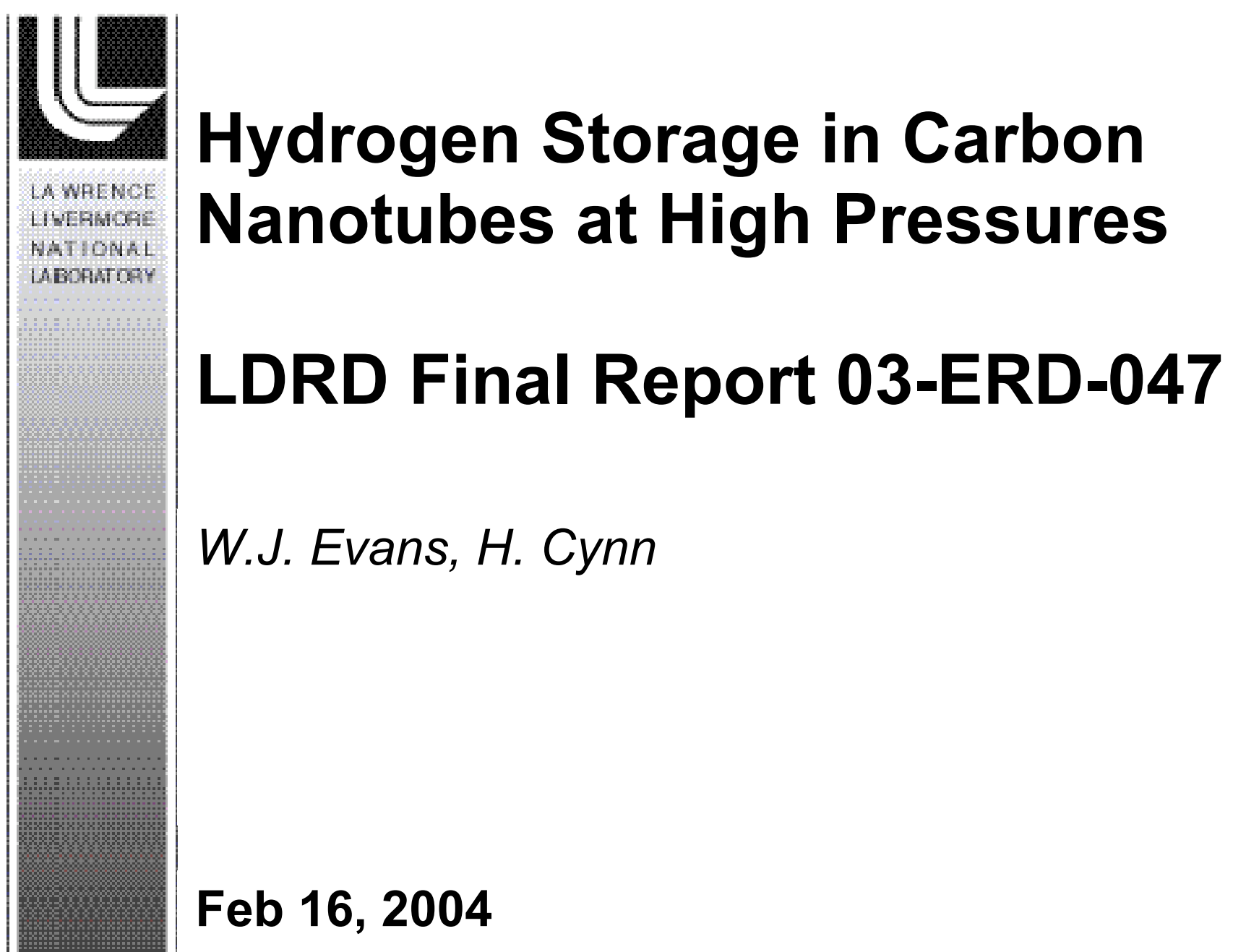




\section{Disclaimer}

This document was prepared as an account of work sponsored by an agency of the United States Government. Neither the United States Government nor the University of California nor any of their employees, makes any warranty, express or implied, or assumes any legal liability or responsibility for the accuracy, completeness, or usefulness of any information, apparatus, product, or process disclosed, or represents that its use would not infringe privately owned rights. Reference herein to any specific commercial product, process, or service by trade name, trademark, manufacturer, or otherwise, does not necessarily constitute or imply its endorsement, recommendation, or favoring by the United States Government or the University of California. The views and opinions of authors expressed herein do not necessarily state or reflect those of the United States Government or the University of California, and shall not be used for advertising or product endorsement purposes.

\section{Auspices Statement}

This research is funded by the Laboratory Directed Research and Development (LDRD) Program at Lawrence Livermore National Laboratory (LLNL) under project (03-ERD-047). The LDRD Program is mandated by Congress to fund laboratory-initiated, long-term research and development (R\&D) projects in support of the DOE and national laboratories' mission areas. The Director's Office LDRD Program at LLNL funds creative and innovative R\&D to ensure the scientific vitality of the Laboratory in mission-related scientific disciplines.

This work was performed under the auspices of the U.S. Department of Energy by University of California, Lawrence Livermore National Laboratory under Contract W-7405-Eng-48. 


\title{
Hydrogen Storage in Carbon Nanotubes at High Pressures LDRD Final Report 03-ERD-047
}

\author{
William J Evans, Hyunchae Cynn \\ H-Division, Condensed Matter Physics \\ Physics and Advanced Technologies Directorate \\ Lawrence Livermore National Laboratory \\ wjevans@1lnl.gov, 925-424-3356
}

This goal of this project was to perform feasibility experiments and measurements of the fundamental interactions between hydrogen and single wall carbon nanotubes (SWNT) at high pressures. High-pressure is an adjustable experimental parameter for tuning interaction strengths, thereby elucidating and providing insights into the fundamental nature of the $\mathrm{H}_{2} / \mathrm{SWNT}$ system. We have developed and utilized systems and methodologies to make $\mathrm{x}$-ray scattering, optical spectroscopic and electrical transport measurements. These activities have been productive in demonstrating capabilities and measuring properties of SWNTs under high-pressure conditions. We have also developed strong cooperative and complementary relationships with academic research colleagues at Stanford University. Building on these results and relationships, we hope to continue and expand our research as co-investigators in a joint Harvard-LLNL-Stanford proposal to the DOE "Grand Challenge" for Basic and Applied Research in Hydrogen Storage (Solicitation \# DE-PS36-03GO93013).

Hydrogen storage is an active research topic with important basic science implications and a crucial enabling technology for advanced energy systems. Measurements of the $\mathrm{H}_{2}$ storage capacity indicate that it may achieve or exceed the storage capacity level (6.5 wt$\%)$ mandated by the DOE hydrogen plan for fielding a hydrogen-fueled vehicle. The $\mathrm{H}_{2} / \mathrm{SWNT}$ system has been the subject of intensive study and controversy regarding this storage capacity, with various conflicting measurements ranging from $1 \mathrm{wt}-\%$ up to values exceeding $7 \mathrm{wt}-\%[1-5]$. The mechanism and details of the hydrogen storage in SWNT systems is poorly understood and several key questions have not been definitively determined including:

- importance of SWNT structural parameters (tube length, diameter, end termination, bundling)

- adsorption sites, mechanism, and binding energy

- optimal storage capacity and conditions

This project sought to demonstrate techniques to address these key issues using highpressure methodologies. Applying high-pressure conditions leads to an enhancement of the interaction between the hydrogen and the SWNTs. We use this capability in combination with optical, $x$-ray and electrical transport diagnostics to study the lattice parameters, vibrational energies, and electrical properties of hydrogen, SWNTs and the 
$\mathrm{H}_{2} / \mathrm{SWNT}$ system. Additionally high-pressure/low-temperature conditions allow us to immerse SWNT samples in liquid hydrogen, thereby maximizing the hydrogen uptake. Specifically, we directed efforts at developing a high-pressure cell for electric transport measurements, a cryostat for loading liquid hydrogen and optical studies SWNTs in highpressure diamond anvil cells (DACs), and x-ray scattering experiments to measure SWNT structural and packing parameters.

SWNTs have a broad range of properties depending on the details of the nanotube wall structure. These differences are manifested in not only different tube diameters, but also differences in electrical (insulting, semiconducting, metallic) and optical properties[6]. Briefly, SWNTs can be thought of as a sheet of hexagonal carbon that is rolled to form a seamless tube and capped at each end with a fullerene hemisphere. Carbon nanotubes are characterized by two parameters, which describe the dimensions of the sheet, as shown in figure 1.
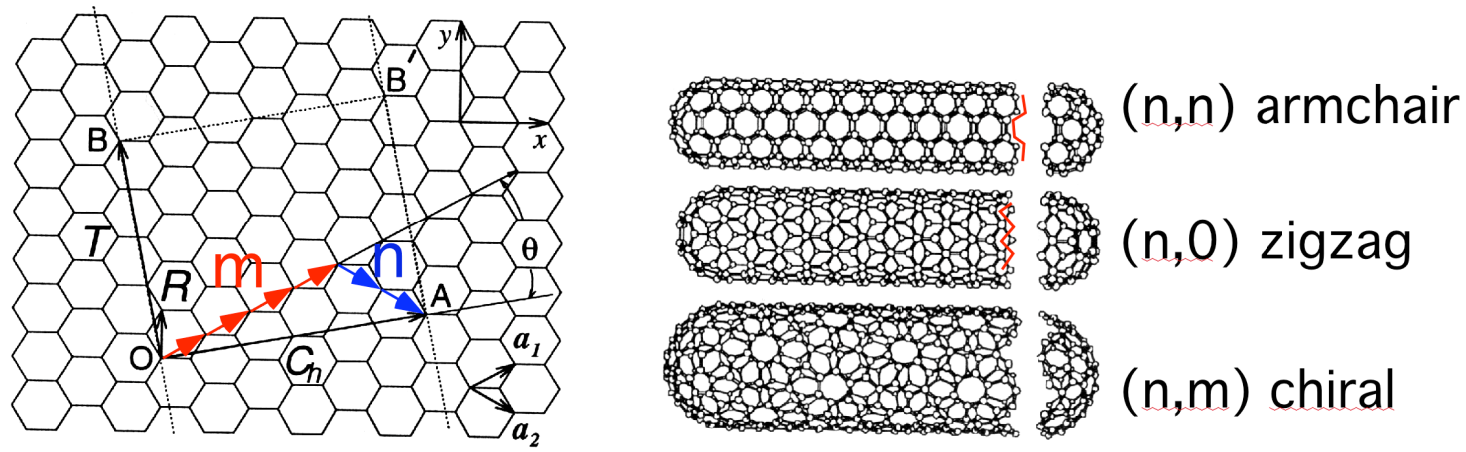

Figure 1. Single Wall Carbon Nanotube parameters. Left: Graphene sheet showing section (OBB'A) to be rolled into tube. $\mathrm{m}, \mathrm{n}$ are the classification parameters. Right: examples of tubes, parameters, and common names for classes of parameters. Images adopted from Saito[6].

Changes in the electrical properties of SWNTs under pressure suggest them as possible sensors. Such measurements also serve as an important test of theoretical modeling capabilities[7]. We developed and used a high-pressure vessel to study these properties. The vessel is operates at pressures of up to 50,000 psi ( 3500 atmospheres), and incorporates electrical feed-throughs for measurements of SWNT-devices under pressure. A schematic diagram and image of the device is shown in figure 2. 

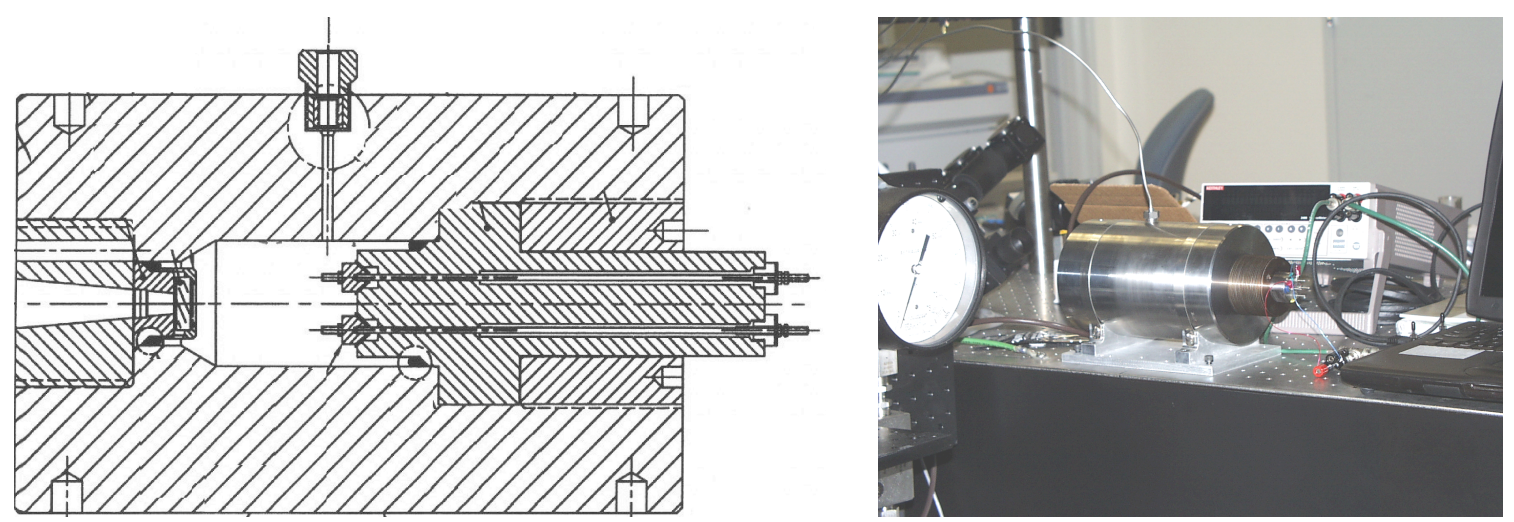

Figure 2. High-Pressure Cell for electrical measurements. Left: Schematic Design. Right: Cell at LLNL in laboratory with associated instrumentation (pressure intensifier, I-V meter, computer control).

Prof. Dai and his research group at the Stanford University Chemistry Dept. are recognized leaders in nanoscience and are able to grow a single carbon nanotube between two electrical contacts. In cooperation with his group we studied the I-V electrical properties of single carbon nanotube devices under pressure. These studies are ongoing, but beyond the importance of pressure, studies include the response to different gases. These studies demonstrate the capability of these single carbon nantoube devices as pressure sensors and can validate theoretical modeling efforts and predictions regarding the pressure-dependent electrical properties of SWNTs.

We made x-ray crystallographic studies of SWNTs in high-pressure DACs using synchroton $\mathrm{x}$-ray scattering techniques. Representative $\mathrm{x}$-ray scattering patterns are shown in figure 3 . Through our experiments and techniques, we were able to measure structural parameters, such as the lattice constants, of SWNT samples. X-ray crystallographic studies of SWNTs are quite sparse because of the low X-ray scattering efficiency of carbon. Our work establishes the ability to measure lattice and structural properties of SWNTs in a DAC. SWNTs are known to crystallize in hexagonal bundles or ropes. Our work confirms and is in good agreement with previous studies[8-10]. Having demonstrated the feasibility of such measurements, we have a strong basis for extending these measurements to high pressures. 


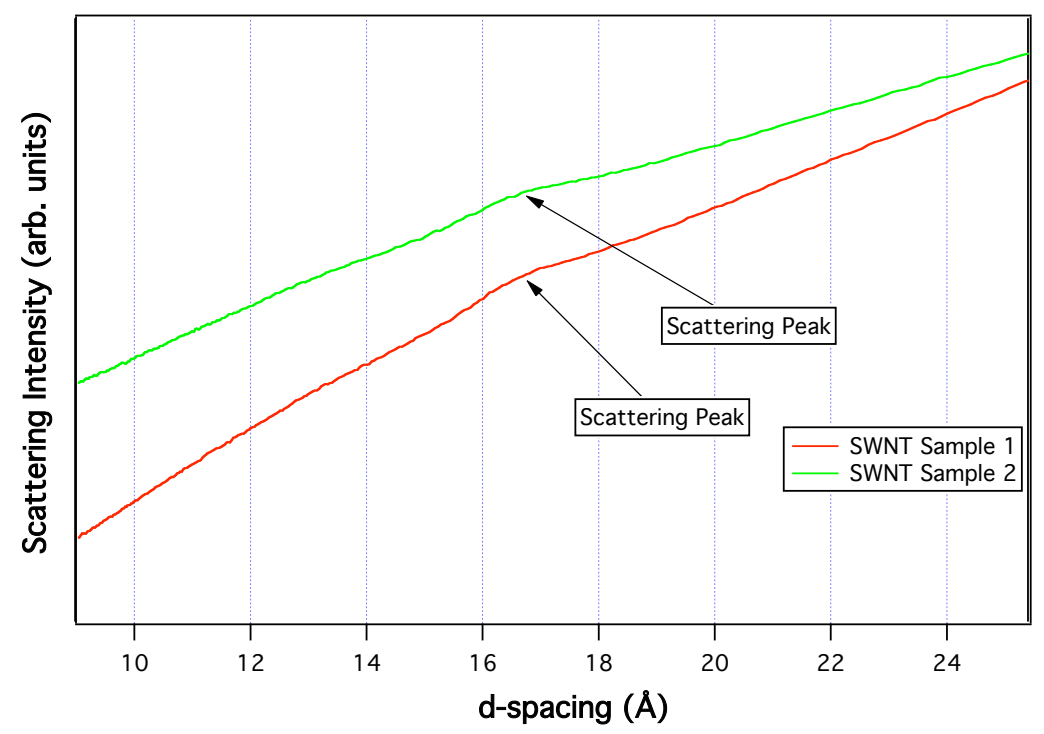

Figure 3. Our X-ray scattering spectra of SWNTs. Peaks are clearly discernible but weak due to technical challenges of $\mathrm{x}$-ray scattering from carbon, a poor scattering low-Z material.

A unique approach of our work is the capability to expose SWNTs to hydrogen at high densities in order to maximize the interaction and uptake. This required development of a cryogenic capability to cool down the cell and load SWNTs in a DAC sample chamber with liquid hydrogen. We implemented this capability and integrated it with an optical spectroscopic measurement system. A photograph of this system is shown in figure 4 .

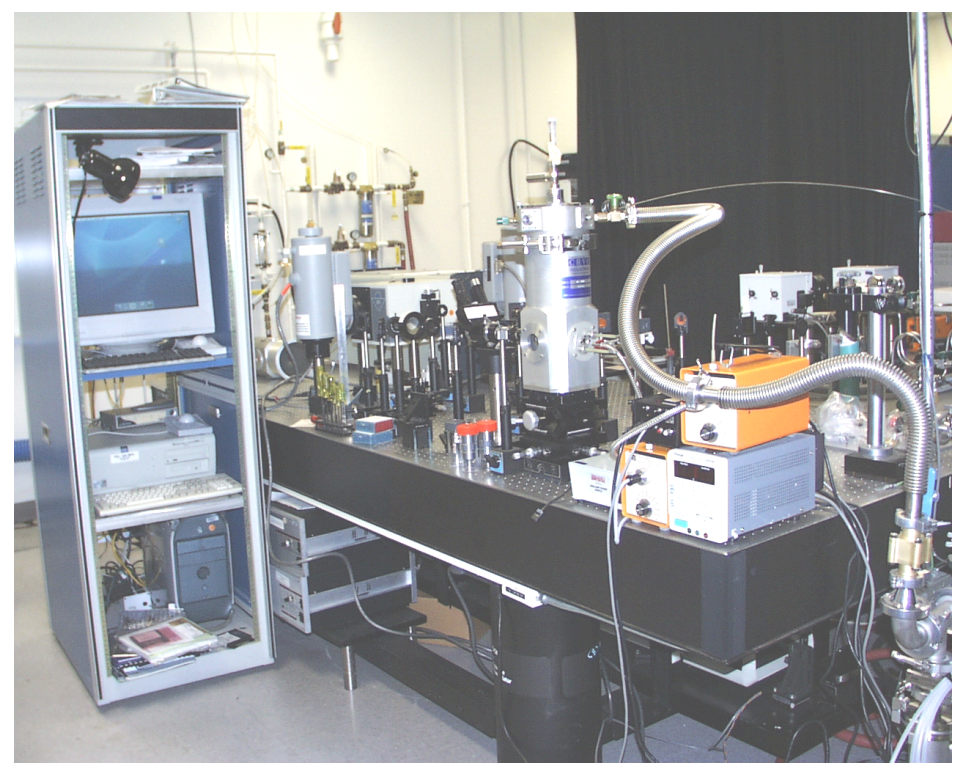

Figure 4. Cryogenic loading system developed and installed at LLNL. Cryostat is the cylindrical silver object near the center. Optical, vacuum and computer control systems are also shown. 
With this system we can measure the pressure and the Raman-active excitations of the SWNTs in the presence of high-density hydrogen. These measurements reflect changes in the molecular environment of the hydrogen and SWNTs, which one would expect if significant binding occurs between these two components. Establishing this capability was a crucial step in demonstrating our capability to perform meaningful and unique studies of the SWNT/Hydrogen system.

The goals of this project have been achieved. We have developed unique capabilities and demonstrated them in the study of SWNTs. These unique capabilities include measurements of the electrical properties of single SWNTs at high pressures and a cryogenic capability for subjecting SWNT to high-density hydrogen. We made measured $\mathrm{X}$-ray diffraction from SWNTs in a DAC and demonstrated our ability to measure structural parameters of these microscopic, low-scattering samples. Our studies of the electrical properties of SWNTs are ongoing and upon completion we plan to publish these results in a peer-reviewed journal. These activities have served us well in demonstrating our capabilities and developing collaborative relationships, which should support our submission of a joint Harvard-LLNL-Stanford proposal to the DOE "Grand Challenge" for Basic and Applied Research in Hydrogen Storage (Solicitation \# DEPS36-03GO93013). 


\section{References}

1. Dillon, A.C., et al., Storage of hydrogen in single-walled carbon nanotubes. Nature, 1997. 386(6623): p. 377-379.

2. Dillon, A.C. and M.J. Heben, Hydrogen storage using carbon adsorbents: past, present and future. Applied Physics A: Mateials Science \& Processing, 2001. 72(2): p. 133-142.

3. Dresselhaus, M.S., K.A. Williams, and P.C. Eklund, Hydrogen Adsorption in Carbon Materials. MRS Bulletin, 1999. 24(11): p. 45-50.

4. Hirscher, M., et al., Are carbon nanostructres an efficient hydrogen storage medium? Journal of Alloy and Compounds, 2003. 356-357: p. 433-437.

5. Züttel, A. and S.-I. Orimo, Hydrogen in Nanostructured, Carbon-Related, and Metallic Materials. MRS Bulletin, 2002. 27(9): p. 705-711.

6. Saito, R., G. Dresselhaus, and M.S. Dresselahus, Physical Properties of Carbon Nanotubes. 1999, London: Imperial College Press. 259.

7. Lu, J.-Q., et al., Metal-to-Semiconductor Transition in Squashed Armchair Carbon Nanotubes. Physical Review Letters, 2003. 90(12): p. 156601-1.

8. Rols, S., et al., Polygonization of single-wall carbon nanotube bundles under high pressure. Physical Review B, 2001. 64: p. 153401.

9. Tang, J., et al., Compressibility and Polygonization of Single-Walled Carbon Nanotubes under Hydroastatic Pressure. Physical Review Letters, 2000. 85(9): p. 1887-1889.

10. Tang, J., et al., Revelaing properties of single-walled carbon nanotubes under high pressure. Journal of Physics: Condensed Matter, 2002. 14: p. 10575-10578. 